\title{
Effective Keyword Search in Relational Databases
}

\author{
Fang Liu, Clement Yu \\ Computer Science Department \\ University of Illinois at Chicago \\ \{fliu1,yu\}@cs.uic.edu
}

\author{
Weiyi Meng \\ Computer Science Department \\ Binghamton University \\ meng@cs.binghamton.edu
}

\author{
Abdur Chowdhury \\ Search \& Navigation Group \\ America Online, Inc. \\ cabdur@aol.com
}

\begin{abstract}
With the amount of available text data in relational databases growing rapidly, the need for ordinary users to search such information is dramatically increasing. Even though the major RDBMSs have provided full-text search capabilities, they still require users to have knowledge of the database schemas and use a structured query language to search information. This search model is complicated for most ordinary users. Inspired by the big success of information retrieval (IR) style keyword search on the web, keyword search in relational databases has recently emerged as a new research topic. The differences between text databases and relational databases result in three new challenges: (1) Answers needed by users are not limited to individual tuples, but results assembled from joining tuples from multiple tables are used to form answers in the form of tuple trees. (2) A single score for each answer (i.e. a tuple tree) is needed to estimate its relevance to a given query. These scores are used to rank the most relevant answers as high as possible. (3) Relational databases have much richer structures than text databases. Existing IR strategies are inadequate in ranking relational outputs. In this paper, we propose a novel IR ranking strategy for effective keyword search. We are the first that conducts comprehensive experiments on search effectiveness using a real world database and a set of keyword queries collected by a major search company. Experimental results show that our strategy is significantly better than existing strategies. Our approach can be used both at the application level and be incorporated into a RDBMS to support keyword-based search in relational databases.
\end{abstract}

\section{INTRODUCTION}

The amount of available structured data (in internet or intranet or even on personal desktops) for ordinary users grows rapidly. Besides data types such as number, date and time, structured databases usually also contain a large amount of text data, such as names of people, organizations and products, titles of books, songs and movies, street addresses, descriptions or reviews of products, contents of papers, and lyrics of songs, etc. The need for ordinary users to find information from text in these databases is dramatically increasing. The objective of this paper is to provide effective search of text information in relational databases. We take a lyrics database (Figure 1) as an example to illustrate the problem. There are five tables in the lyrics database. Table Artist has one text column: Name. Table Album has one text column: Title. Table Song has two text columns: Title and Lyrics. The tuples of Table Artist and those of Table Album have m:n relationships (an album may be produced by multiple artists and

Permission to make digital or hard copies of all or part of this work for personal or classroom use is granted without fee provided that copies are not made or distributed for profit or commercial advantage and that copies bear this notice and the full citation on the first page. To copy otherwise, or republish, to post on servers or to redistribute to lists, requires prior specific permission and/or a fee.

SIGMOD 2006, June 27-29, 2006, Chicago, Illinois, USA

Copyright 2006 ACM 1-59593-256-9/06/0006 ..\$5.00 an artist may produce more than one album), and Table AritstAlbum is the corresponding relationship table. Table Song-Album is also a relationship table capturing the $m: n$ relationships between tuples of Album and Song (a song may be contained in multiple albums and an album many contain more than one song). Note that Table Aritst-Album and Table Aritst-Album do not have other columns except their primary keys and foreign keys.

\begin{tabular}{|c|c|c|c|c|}
\hline \multicolumn{2}{|c|}{ Artist (A) } & \multicolumn{3}{|c|}{ Artist-Album (AB) } \\
\hline ArtistID & Name & AB-ID & ArtistID & AlbumID \\
\hline a1 & D12 & $\mathrm{ab1}$ & a1 & b1 \\
\hline $\mathrm{a} 2$ & Eminem & $a b 2$ & $\mathrm{a} 2$ & b1 \\
\hline a3 & Code Red & $a b 3$ & $\mathrm{a} 4$ & b3 \\
\hline $\mathrm{a} 4$ & jojo & & & \\
\hline \multicolumn{2}{|c|}{ Album (B) } & \multicolumn{3}{|c|}{ Album-Song (BS) } \\
\hline \begin{tabular}{|l|} 
AlbumID \\
\end{tabular} & Title & \begin{tabular}{|l|} 
BS-ID \\
\end{tabular} & AlbumID & SongID \\
\hline b1 & D12 World & bs1 & b1 & s1 \\
\hline b2 & Off the Wall & bs2 & $\mathrm{b} 2$ & s2 \\
\hline b3 & jojo & bs3 & b3 & s3 \\
\hline \multicolumn{5}{|l|}{ Song (S) } \\
\hline \begin{tabular}{|l|} 
SongID \\
\end{tabular} & Title & \multicolumn{3}{|l|}{ Lyrics } \\
\hline s1 & How Come & \multicolumn{3}{|c|}{ How come we don't ... by... more.. } \\
\hline $\mathrm{s} 2$ & The Kids & \multicolumn{3}{|c|}{ And everyone should get along ... } \\
\hline $\mathrm{s} 3$ & Leave(Get out) & \multicolumn{3}{|c|}{ I've been waiting all day... } \\
\hline
\end{tabular}

Figure 1: Lyrics Database Example

The traditional search model in relational databases requires users to have knowledge of the database schema and to use a structured query language such as SQL or QBE-based interfaces. Even though most of the major RDBMSs have integrated full-text search capabilities using relevance-based ranking strategies developed in information retrieval (IR), they still have the above two requirements for users. Suppose a user is looking for albums titled "off the wall" and he/she cannot remember the exact title. A typical SQL query is shown in Figure 2 and the tuple $b 2$ is expected to be one of the top ranked results. Obviously, this model of search is too complicated for ordinary users.

Select * from Album B

Where Contains (B.title, 'off wall', 1) >0

Order by score(1) desc

Figure 2: An Oracle SQL Example

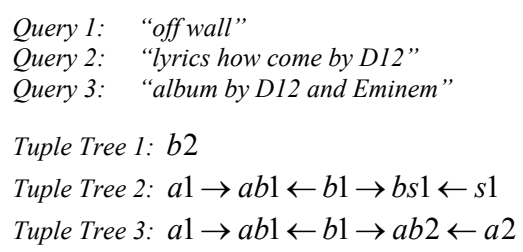

Figure 3: Queries and Tuple Trees

With the tremendous success of web search engines, keyword search has become the most popular search model for ordinary 
users. Users do not need to know the database schema or use a structured query language. Instead, they submit a list of keywords using a very simple interface, and a search engine returns ranked documents based on relevance to the query from its text databases. Therefore, it is extremely desirable to support the keyword search model in relational databases. With such support, a user can avoid writing a SQL query; and he/she can just submit a simple keyword query "off wall" to the lyrics database.

Applying the keyword search techniques in text databases (IR) to relational databases (DB) is a challenging task because the two types of databases are different. First, in text databases, the basic information units searched by users are documents. For a given keyword query, IR systems compute a numeric score for each document and rank the documents by this score. The top ranked documents are returned as answers. In relational databases, however, information is stored in the form of columns, tables and primary key to foreign key relationships. The logical unit of answers needed by users is not limited to an individual column value or even an individual tuple; it may be multiple tuples joined together. Consider Query 1 as shown in Figure 3. Tuple Tree 1 is one desired answer (though the query and the text value are not exactly matched). Note that, in Figure 3, an edge from $a 1$ to $a b 1$ denotes a primary key to foreign key relationship. For Query 2, one desired answer is a joining tree of five tuples (Tuple Tree 2 as shown in Figure 3). Note that the query matches both text columns (title and lyrics) values in the tuple s1. For Query 3, one desired answer is a joining tree of five tuples (Tuple Tree 3 ). Second, effectiveness is a key factor for the success of keyword search. Even in a medium sized database, there may be dozens of candidate answers for an ordinary keyword query. Consider the query "off wall" again. There exists many titles of songs, titles of albums or lyrics of songs that contain both keywords "off" and "wall". Therefore, there are many tuple trees that can be answers for the query. However, these answers are not equally useful to the user. We need to rank the more relevant answers higher. Otherwise, users will be discouraged to use keyword search. Note that a key reason behind the tremendous success of web search engines is that their ranking strategies are highly effective that, for many queries, they can rank relevant web pages in the first ten results out of billions of web pages. Due to the difference in the basic answer unit between document searches and database searches, in relational databases, we need to assign a single ranking score for each tuple tree, which may consist of multiple tuples with text columns, in order to rank the answers effectively. The characteristics of text columns are usually diverse. For example, some text columns such as people's names and album titles are very short, while other text columns such as song lyrics are much longer. In contrast, in text databases, we only need to compute a score for each single document. Thus, the ranking strategy for relational databases needs to consider more factors and is more complicated. Third, relational databases have much richer structures than text databases. For a given query, we desire answers to be returned with semantics. For example, for Query 2 and one answer Tuple Tree 2, it is quite obvious that the subquery "how come" is the Song title of the tuple $s 1$, and the subquery "D12" is the Artist name of the tuple al, although all individual keywords (except "D12") in the two queries are very common words, and some individual keywords also appear in other text values of Tuple Tree 2 (e.g., "D12" appears in the title of $b 1$, and "how" and "come" appear in lyrics of $s 1)$. We consider the correspondences between sub-queries in a given query and database columns in an answer as the semantics of the query in the answer. In summary, in relational databases, we have three key steps for processing a given keyword query. (1) Generate all candidate answers, each of which is a tuple tree by joining tuples from multiple tables. (2) Then compute a single score for each answer. The scores should be defined in such a way so that the most relevant answers are ranked as high as possible. (3) And finally return answers with semantics.

Recently, keyword search on relational databases has merged. DBXplorer [1], DISCOVER [10], BANKS [2], and Hristidis et al. [11] are systems that support keyword search on relational databases. For the first step, they generate tuple trees from multiple tables as answers. The first three systems require an answer containing all keywords in a query, while the last one only requires an answer containing some but not necessarily all keywords in the query. Efficiency has been the focus for the first step [1, 2 10, 11]: rules are designed to avoid generation of unnecessary tuple trees, and more efficient algorithms are proposed to improve the time and space complexities. For the second step, the first two systems use a very simple ranking strategy: the answers are ranked in ascending order of the number of joins involved in the tuple trees. When two tuple trees have the same number of joins, their ranks are determined arbitrarily. Thus, all tuple trees consisting of a single tuple are ranked ahead of all tuples trees with joins. The ranking strategy of the BANKS system is to combine two types of information in a tuple tree to compute a score for ranking: a weight (similar to PageRank for web pages) of each tuple, and a weight of each edge in the tuple tree that measures how related the two tuples are. The strategy of DBXplorer [1] and DISCOVER [10] and the strategy of BANKS [2] for the second step do not utilize any state-of-the-art IR ranking methods, which have been tremendously successful. In a database that contains a large amount of text data, these strategies will be shown not to work well. Hristidis et al. [11] propose a strategy by applying IR-style ranking methods into the computation of ranking scores in a straightforward manner. They consider each text column as a collection and each value in the text column as a document. A state-of-the-art IR ranking method is used to compute a score between a given query and each text column value in the tuple tree. A final score is obtained by dividing the sum of all these scores by the number of tuples (i.e. the number of joins plus 1) in the tree. However, they only concentrate on the efficiency issue of the implementation of the ranking strategy and do not conduct any experiments on the effectiveness issue. In addition, our experimental results show that their strategy ignores some important factors that are critical for search effectiveness.

Our paper focuses on search effectiveness. We propose a novel ranking strategy that ranks answers (tuple trees) effectively and returns answers with basic semantics. This strategy can be used both at the application level and be incorporated into a RDBMS to support keyword search in relational databases. We adapt the framework proposed in Hristidis et al. [11] to generate tuple trees as answers for a given query. Efficiency issues are not investigated in this paper. We emphasize that effectiveness of keyword search of text data (in both text and structured databases) is at least as important as efficiency.

Our key contributions are as follows: 
1. We identify four new factors that are critical to the problem of search effectiveness in relational databases.

2. We propose a novel ranking strategy to solve the effectiveness problem. Answers are returned with basic semantics.

3. We are the first to conduct comprehensive experiments for the effectiveness problem.

4. Experimental results show that our strategy is significantly better than existing works in effectiveness $(77.4 \%$ better than [11] and $16.3 \%$ better than Google ${ }^{1}$ ).

In Section 2, we briefly introduce the framework for generation of answers (tuple trees) for keyword search in relational databases. In Section 3, we briefly introduce the background of a recent IR similarity function. In Section 4, we analyze new challenges for keyword search in relational databases, identify new factors that are critical to ranking effectiveness, and propose a novel ranking strategy. In Section 5, we present experimental results to demonstrate the effectiveness of our ranking strategy. Section 6 discusses related works. Section 7 draws the conclusion and outlines directions for future work.

\section{ANSWER GENERATION}

In this section, we describe the framework for generating answers for given keyword queries (a modification of [11]). Section 2.1 describes what an answer is for a keyword query in relational databases. Section 2.2 gives an algorithm to generate answers.

\subsection{Tuple Trees As Answers}

We use a graph to model a database schema. A schema graph is a directed graph $S G$. For each table $R_{i}$ in the database, there is a node in the schema graph. If there is a primary key to foreign key relationship from the table $R_{i}$ to the table $R_{j}$ in the database, then there is an edge from the node $R_{i}$ to the node $R_{j}$ in the schema graph. Each table $R_{i}$ has $m_{i}\left(m_{i}>=0\right)$ text columns $\left\{c_{1}^{i}, c_{2}^{i}, \ldots, c_{m i}^{i}\right\}$. Figure 4 shows a schema graph of the lyrics database (columns are shown in Figure 1). A tuple tree $T$ is a joining tree of tuples. Each node $t_{i}$ in $T$ is a tuple in the database. Suppose $\left(R_{i}, R_{j}\right)$ is an edge in the schema graph. Let $t_{i} \in R_{i}, t_{j} \in R_{j}$, and $\left(t_{i}\right.$ join $\left.t_{j}\right) \in\left(R_{i}\right.$ join $\left.R_{j}\right)$. Then $\left(t_{i}, t_{j}\right)$ is an edge in the tuple tree $T$. The size of a tuple tree $T$ is the number of tuples involved. Note that a single tuple is the simplest tuple tree with size 1 . A keyword query $Q$ consists of a list of keywords $\left\{k_{1}, k_{2}, \ldots k_{n}\right\}$. For a given query $Q$, an answer must be a tuple tree $T$ that satisfies the following conditions: (1) every leaf node $t_{i}$ in $T$ contains at least one keyword in $Q$ (more precisely, at least a text column value of the tuple $t_{i}$ contains at least one keyword in $Q$ and different leaf nodes may contain the same keyword), and (2) each tuple only appears at most once in the tuple tree. Note that a non-leaf node (a node has two or more edges) may or may not contain any keyword. This definition implies that (a) if an answer contains multiple tuples, they must be joined together as a tree, (b) we assume the OR semantics (i.e. an answer must contain at least one query keyword) for answering a query, and (c) there is no redundancy, because if we remove any leaf node, we will lose the corresponding keyword match between the query and the node, and if we remove any non-leaf node, the tuple tree becomes

\footnotetext{
${ }^{1}$ Although Google retrieves from text databases (not from relational databases), its big success necessitates a comparison.
}

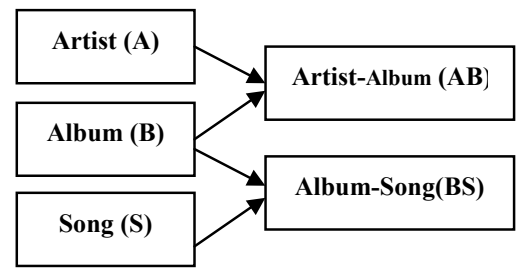

Figure 4: The Lyrics Database Schema Graph

$$
\begin{aligned}
& a 1 \rightarrow a b 1 \leftarrow b 1 \rightarrow b s 1 \leftarrow s 1 \\
& a 1 \rightarrow a b 1 \leftarrow b 1 \rightarrow a b 2 \leftarrow a 2 \\
& A^{\mathrm{Q}} \rightarrow A B^{F} \leftarrow B^{F} \rightarrow B S^{F} \leftarrow S^{\mathrm{Q}} \\
& A^{Q} \text { join } A B^{F} \text { on }\left(A^{Q} \text {.ArtistID }=A B^{F} \text {. ArtistID) join } B^{F}\right. \text { on } \\
& \left(A B ^ { F } \text { .AlbumID } = B ^ { F } \text { .AlbumID) join } B S ^ { F } \text { on } \left(B^{F}\right.\right. \text {.AlbumID = } \\
& B S^{F} \text {.AlbumID) join } S^{Q} \text { on }\left(B S^{F} \text {.SongID }=S^{Q} \text {. SongID }\right) \\
& A^{1, \mathrm{Q}} \rightarrow A B^{1, F} \leftarrow B^{F} \rightarrow A B^{2, F} \leftarrow A^{2, \mathrm{Q}} \\
& \text { (Answer Graph 3) } \\
& \text {...join.. Where }\left(A^{1, Q} \text {.ArtistID } !=A^{2, Q}\right. \text {.ArtistID) and } \\
& \left(A B^{l, F} A B^{l, F} \mathrm{AB}-\mathrm{ID} !=A B^{l, F}\right. \text {.AB-ID) }
\end{aligned}
$$

Figure 5: Tuple Trees, Answer Graphs, Join Expressions

disconnected. Take Tuple Tree 2 and Tuple Tree 3 in Section 1 as examples (also shown in Figure 5). In Tuple Tree 2, the leaf nodes al contains the keyword "D12", and sl contains two keywords "how" and "come" in Query 2. In Tuple Tree 3, leaf nodes $a 1$ and $a 2$ contain the keywords "D12" and "Eminem" in Query 3 respectively; the non-leaf node $b 1$ also contains the keyword "D12". Note that we consider a tuple tree as an answer as long as it satisfies the definition. An answer may or may not be relevant to a given query.

\subsection{Answer Graph to Generate Answers}

In this section, we briefly describe an algorithm (a modification of the algorithm described in [11]) to generate tuple trees as answers. For a given keyword query $Q$, the query tuple set $R^{Q}$ of a table $R$ is defined as the set of all tuples in $R$ that contain at least one keyword in $Q$. For example, the query tuple sets of Table Artist for Query 1, Query 2 and Query 3 are $A^{Q 1}=\{\}, A^{Q 2}=\{a 1\}$ and $A^{Q 3}=\{a 1, a 2\}$ respectively. We define the free tuple set $R^{F}$ of a table $R$ as the set of all tuples in $R$. For example, the free query set for Table Artist is $A^{F}=\{a 1, a 2, a 3\}$. Implied from the definition of an answer, for a given query $Q$, if a tuple tree $T$ is an answer, then each leaf node $t_{i}$ in Table $R_{i}$ belongs to the query tuple set $R_{i}^{Q}$, and each non-leaf node $t_{j}$ in Table $R_{j}$ belongs to the free tuple set $R_{j}^{F}$. We use $R^{Q o r F}$ to denote a tuple set, which may be either a query tuple set or a free tuple set. Due to the existence of $m: n$ relationships (for example, an album may be produced by more than one artist), tuple sets of the same table may appear more than once in a join expression. If this happens, each occurrence of the same tuple set is considered to be a different alias of the tuple set. Now, we define an answer graph as a join expression on alias of tuple sets that produces tuple trees as answers. An answer graph is a directed graph $A G$ of alias of tuple sets, where each leaf node $R_{i}^{x, Q}$ is the $x$-th alias of the query tuple set $R_{i}^{Q}$ of the table $R_{i}$ and each non-leaf node $R_{j}^{y, F}$ is the $y$-th alias of the free tuple set $R_{j}^{F}$ of the table $R_{j}$, and each edge $\left(R_{i}^{x, Q o r F}, R_{j}^{y, Q o r F}\right)$ corresponds to an edge $\left(R_{i}, R_{j}\right)$ in the schema graph $S G$ and this edge also corresponds to a join clause " $R_{i}^{x, Q o r F}$ join $R_{j}^{y, Q o r F}$ on $R_{i}^{x, Q o r F}$.PrimaryKey $=R_{j}^{y, Q o r F}$.ForeignKey". We define the size of an answer graph as the number of nodes. Obviously, the size of an answer graph is the same as that of a tuple tree it produces. For example, the Answer Graph 2 and Answer Graph 3 (as well as the 
Input: query $Q$, schema graph $S G, \operatorname{maxn}, M A X N$

Output: a set of answer graphs $S$

1. Generate all non-empty query tuple sets $\left\{R_{1}{ }^{Q}, R_{2}{ }^{Q}, \ldots R_{n}{ }^{Q}\right\}$ and all nonempty free tuple sets $\left\{R_{1}{ }^{F}, R_{2}{ }^{F}, \ldots R_{n}{ }^{F}\right\}$ for $Q$. Add the two sets of tuple sets into a queue $E$. Note that, in the following steps, these tuple sets are considered as symbols.

2. While $E$ is not empty \{

Pop the head $h$ from $E$.

If $h$ violates the constraints of MAXN and $\operatorname{maxn}$, then $h$ is discarded

Else

If $h$ is a valid answer graph ${ }^{+}$, then add it to $S$.

For each $R_{i}^{\mathrm{Q} \text { or F }}$ in $h$

For each $R_{j}$ that is adjacent to $R_{i}$ in $\mathrm{SG}\{$

Add $R_{j}^{\mathrm{Q}}$ with an edge into $h$ to get a new $h l$.

Add $R_{j}^{\mathrm{F}}$ with an edge into $h$ to get a new $h 2$.

If $h l$ is not in $E$, then push it into the end of $E$.

If $h 2$ is not in $E$, then push it into the end of $E$.$$
\text { \} }
$$

\}

3. For each graph of tuple sets in $S$, if there is more than one occurrence for any tuple set, give these occurrences different alias names.

4. Return $S$.

${ }^{+}$Note that whether an $h$ is a valid answer graph is determined by the definition of an answer graph, as well as the two parameters maxn and $M A X N$.

Figure 6: Algorithm for Answer Graph Generation

corresponding join expressions) that generate Tuple Tree 2 and Tuple Tree 3 are shown in Figure 5 (If there is only one occurrence of a tuple set, we omit the alias number). In Answer Graph 3, there are two alias of tuple sets $A^{1, Q}$ and $A^{2, Q}$ for the same query tuple set $A^{Q}$ of Table $A$. If there exists more than one alias for a tuple set, in the corresponding join expression, we need to add a condition such as " $A^{1, Q}$.ArtistID $!=A^{2, Q}$.ArtistID" to avoid producing tuple trees such as $a 1 \rightarrow a b 1 \leftarrow b 1 \rightarrow a b 1 \leftarrow a 1$, which violates the second condition of the definition of an answer. If there is an edge $\left(R_{i}, R_{j}\right)$ in the schema graph $S G$, and $n$ is the maximum number of distinct tuples in the foreign table $R_{j}$ that can be joined with one tuple in the primary table $R_{i}$, then, in theory, each tuple set of $R_{i}$ may connect to $n$ tuple sets of $R_{j}$ in an answer graph in order to produce all possible tuples trees, each of which contains one tuple of $R_{i}$ that is joined with at most $n$ distinct tuples of $R_{j}$. And the number of answer graphs is only data bounded by the query and the database. Thus, we set up two parameters, maxn (for each tuple set of a primary table, it is the maximum number of tuple sets of a foreign table that can be joined) and $M A X N$ (the maximum number of tuple sets in an answer graph) to avoid generating complicated but less meaningful answer graphs. Note that, for a given query, there is usually more than one answer graph. For example, besides Answer Graph 2, $A^{Q^{2}}$ and $S^{Q^{2}}\left(S^{Q 2}=\{\mathrm{s} 1\}\right)$ are also two answer graphs for Query 2. Figure 6 gives a breadth-first search algorithm to generate all answer graphs $A G s$ for a given query $Q$ and a given schema graph $S G$. With answer graphs, it is rather straightforward to produce tuple trees as answers by evaluating the corresponding join expressions. It can be proved that the answer graphs output by our algorithm can produce all and only all answers (tuple trees) if we do not apply the constraints of maxn and $M A X N$.

In summary, in order to generate answers for a given query, the system first finds all tuple sets, then it generates all answer graphs using the tuple sets and the schema graph, and finally, it produces all tuple trees as answers by evaluating the join expressions in the answer graphs. The efficiency issue of answer generation is not the focus of this paper and will not be discussed.

\section{BACKGROUND IN IR RANKING}

Modern IR systems rank documents for a given query. The higher a document is ranked, the more likely the document is relevant or useful to the query. In Section 3.1 we describe how the effectiveness of IR ranking is evaluated. The IR effectiveness measures will be used in our experiments. In Section 3.2, we introduce and discuss a recent IR similarity function and analyze some critical factors that affect search effectiveness.

\subsection{Effectiveness Measures in IR}

In IR, there are many measures to evaluate effectiveness. 11point precision and recall (precision is the number of relevant documents retrieved divided by the number of retrieved documents, and recall is the number of relevant documents retrieved divided by the number of relevant documents) is a standard measure. At each of the 11 recall levels $(0,0.1,0.2, \ldots, 1)$, a precision value is computed. These 11 precisions are usually plotted in a graph to illustrate the overall effectiveness as well as the trade off between precision and recall. Mean average precision (MAP) is another standard measure. A precision is computed after each relevant document is retrieved. Then we average all precision values to get a single number to measure the overall effectiveness.

For ranking tasks in which users look for a single or a very small set of target documents (such as homepage search and question answering [19]) in a large collection, the reciprocal rank is another popular measure. For a given query, the reciprocal rank is 1 divided by the rank at which the first correct answer is returned or 0 if no correct answer is returned. For example, if the first relevant document is ranked at 5 , then the reciprocal rank is $1 / 5$.

In IR experimentation, values of each of the above measures are usually averaged over a set of queries to get a single number to evaluate the effectiveness of an IR system. Note that "evaluation of search effectiveness has been a cornerstone of $I R$ " [18], and effectiveness is one of the two (efficiency is the other one) most important problems in IR. This paper is the first work that conducts a comprehensive effectiveness evaluation on the problem of keyword search in relational databases.

\subsection{Ranking Model in IR}

To rank documents, IR systems assign a score for each document as an estimation of the document relevance to the given query. The widely used model to compute such a score is the vector space model [26]. Each text (both documents and queries) is represented as a vector of terms, each of which may be an individual keyword or a multi-word phrase. The vocabulary of terms makes up a term space. Each term occupies a dimension in the space. Each text (a document or a query) is represented as a vector on this term space, and each item in the vector of a text has a non-negative weight, which measures the importance of the corresponding term $k$ in the text. Thus, a similarity value between a document vector $D$ and a query vector $Q$ can be computed as the ranking score.

Formula 1 shows the inner product (dot product) function to compute the similarity. Weighting a term in a document (the weight $(k, D)$ component in Formula 1) is the most critical problem in computing similarity values. Formula 2 shows the 


$$
\begin{aligned}
& \operatorname{Sim}(Q, D)=\sum_{k \in Q, D} \text { weight }(k, Q) * \text { weight }(k, D) \\
& \text { weight }(k, D)=\frac{n t f}{n d l} * i d f \\
& n t f=1+\ln (1+\ln (t f)) \\
& \qquad i d f=\ln \frac{N}{d f+1} \\
& n d l=(1-s)+s * \frac{d l}{a v g d l}
\end{aligned}
$$

pivoted normalization weighting method $[17,18]$, which is one of the most widely used weighting methods in IR. Note that, conceptually, we put the idf component into Formula 2 but not into Formula 1. The weight of a term in a document is determined by the following three factors.

- Term Frequency ( $t f$ in Formula 2.1): the number of occurrences of a term in a document. Intuitively, the more a term occurs in a document, the higher the weight of the term should be. However, the same term may occur many times in a long document, and the importance of a term should not be linearly dependent on the raw $t f$ when $t f$ is rather large. It has been accepted in IR that the raw $t f$ should be dampened. Formula 2.1 applies the $\log$ function twice to normalize the raw $t f$ to get $n t f$.

- Document Frequency ( $d f$ in Formula 2.2): the number of documents that a term occurs in a collection. By intuition, in a collection, the more documents a term appears in, the worse discriminator it is, and it should be assigned a smaller weight. Formula 2.2 shows the inverse document frequency (idf) weighting method to normalize $d f$ : dividing the total number of documents ( $N$ in Formula 2.2) by $(d f+1)$ and then applying the $\log$ function

- Document Length ( $d l$ in Formula 2.3): the length of a document in bytes or in number of terms contained in the document. Because longer documents contain more terms and higher term frequencies, longer documents tend to have higher inner product values for a given query. Formula 2.3 provides a normalization to reduce the term weights in long documents, where avgdl is the average document length in the collection, and $s$ is a constant and is usually set to 0.2 .

Weighting a term in a query (the weight $(k, Q)$ component in Formula 1) is rather simple: We use raw term frequency (qtf) in the query. Note that normalization on the above three factors has significantly improved search effectiveness in IR than the simple $t f^{*} i d f$ weighting methods [18].

\section{NOVEL RANKING STRATEGY FOR RELATIONAL DATABASES}

In this section, we propose a novel ranking strategy for effective keyword search in relational databases. In IR, a document is a basic information unit stored in a text database; and it is also the basic unit of answers needed by users. A similarity value between a given query and a document is computed to rank documents. However, the basic text information unit stored in a relational database is a text column value, while the basic unit of answers needed by users is a tuple tree, which is assembled by joining multiple tuples, each of which may contain zero, one or multiple text column values (each text column value is considered as a document). A similarity value between a given query and a tuple

$$
\operatorname{Sim}(Q, T)=\sum_{k \in Q, T} \text { weight }(k, Q) * \text { weight }(k, T)
$$

tree needs to be computed to rank tuple trees. This value has two factors: similarity contributed from each text column value in the tuple tree, and a combination of all these contributions. Let $T$ be a tuple tree and $\left\{D_{1}, D_{2}, \ldots, D_{m}\right\}$ be all text column values in $T$. We define each text column value $D_{i}$ as a document and $T$ as a super-document. Then we can compute a similarity value between the query $Q$ and the super-document $T$ as shown in Formula 3 to rank tuple trees. The similarity is the dot product of the query vector and the super-document vector. The method of weighting a term in the query, weight $(k, Q)$, still uses the term's raw qtf (term frequency in the query). Our focus is on weight(k,T), the weight of a term $k$ in a super-document $T$.

In Section 4.1, we introduce the ranking strategy proposed by Hristidis et al. [11]. In Section 4.2, we identify four important factors that affect search effectiveness and propose a novel term weighting strategy. Section 4.3 identifies the schema term problem and proposes a solution. Section 4.4 proposes phrasebased and concept-based search models that improve effectiveness and can return semantics.

\subsection{Ranking Strategy in Related Work}

The weighting method in Hristidis et al. [11] considers each text column as a collection, and uses the standard IR weighting method as shown in Formula 2 to compute a weight for each term $k$ in each document $D_{i}$. Then, as shown in Formula 4, each weight

$$
\text { weight }(k, T)=\sum_{D_{i} \in T} \text { weight }\left(k, D_{i}\right) / \operatorname{size}(T)
$$

is normalized (divided by size(T), i.e. the number of tuples in $T$ ). The weights of the term in all documents are summed to obtain the term weight in the super-document $T$. Formula 4 identifies and deals with a new factor, size $(T)$, that affects similarity. However, more factors need to be considered.

\subsection{Four Normalizations}

We identify four important factors that affect search effectiveness and propose a novel term weighting strategy as shown by Formulas 5.1 and 5.2. Formula 5.1 computes a term's weight in a document $D_{i}$, and Formula 5.2 computes the same term's weight

$$
\begin{aligned}
& \text { weight }\left(k, D_{i}\right)=\frac{n t f^{*} i d f^{g}}{n d l * N \operatorname{size}(T)} \\
& \text { weight }(k, T)=\operatorname{Comb}\left(\operatorname{weight}\left(k, D_{1}\right), \ldots, \operatorname{weight}\left(k, D_{m}\right)\right)
\end{aligned}
$$

in the tuple tree $T . n t f$ is still computed using Formula 2.1; Nsize(T) is a new tuple tree normalization factor (see Section 4.2.1 and Formula 6); $n d l$ is a new document length normalization factor (Section 4.2.2 and Formula 7); $i d f^{g}$ is a new inverted document frequency weight (Section 4.2.3 and Formula 8); $\operatorname{Comb}($ ) is a new function to combine term weights in documents into a term weight in a tuple tree (Section 4.2.4 and Formula 9).

\subsubsection{Tuple Tree Size Normalization}

The tuple tree size factor, size(T), is similar to the document length $(d l)$ factor discussed in Section 3.2 in the following sense: a tuple tree with more tuples tends to contain more terms and higher term frequencies. However, using the raw size $(T)$ as shown in Formula 4 can be sub-optimal, especially for a complex query whose relevant answers are tuple trees involving multiple tuples, 
each of which contains a subset of the query keywords. Consider the 26th query $Q$ ("jojo leave lyrics") in the appendix and the example in Figure 1. A relevant answer is a tuple tree $b 3 \rightarrow b s 3 \leftarrow s 3$. Let us call it $T_{3}$. Let $T 4$ be the single-node tuple tree that only contains the tuple $b 3$, which contains the keyword "jojo", and T5 be the single-node tuple tree that only contains the tuple $s 3$, which contains the keyword "leave". Both $T_{4}$ and $T_{5}$ are answers for the query under OR semantics, but they are not as relevant as $T_{3}$ to the query. Suppose weight ("jojo",b3) $=w 1$ and weight ("leave",s3) $=w 2$ (all other weights are zeros). Then $\operatorname{Sim}\left(Q, T_{4}\right)=w 1, \operatorname{Sim}\left(Q, T_{5}\right)=w 2$ and $\operatorname{Sim}\left(Q, T_{3}\right)=(w 1+w 2) / 3$. Obviously, at least one of $T_{4}$ and $T_{5}$ will be ranked ahead of $T_{3}$ (if we ignore the tie situation), and this ranking is ineffective because $T_{3}$ should be ranked higher than both $T_{4}$ and $T_{5}$. Thus, size(T) should be normalized. We borrow the Formula 2.3 for document length normalization to tuple tree size normalization: the weight of a term in a document is divided by $N \operatorname{size}(T)$ as shown in Formula 5.1 and Formula 6 instead of the raw size(T). For

$$
\operatorname{Nsize}(T)=(1-s)+s * \frac{\operatorname{size}(T)}{\text { avgsize }}
$$

document length normalization, the avgdl is averaged on all documents in the collection. Thus, one way to compute avgsize is to generate all possible answer graphs using the algorithm described in Figure 6 by using a pseudo query $Q_{p}$ and assuming all query tuple sets and all free tuple sets are non-empty, and then average the sizes of all the answer graphs With this normalization, the new similarity values for $T_{3}, T_{4}$ and $T_{5}$ become $w 1, w 2$ and $(w 1+w 2) / 1.15$ respectively. As a result, using $N \operatorname{size}(T), T_{5}$ is much more likely to be ranked ahead of both $T_{3}$ and $T_{4}$ than using the raw $\operatorname{size}(T)$. Note that this normalization is independent of any keyword in the query and any document in the tuple tree.

\subsubsection{Document Length Normalization Reconsidered}

When we logically combine multiple documents into one superdocument $T$, the document length factor needs to be reconsidered because collections (i.e. text columns) have their own avgdl values, which may be very different. Take Query 2 as an example. In the answer Tuple Tree 2, the keywords "how" and "come" occur in both $D_{\text {Title }}$ (i.e. the Title column value in the tuple $s 1$ ) and $D_{\text {Lyrics }}$ (i.e. the Lyrics column value in the tuple $s 1$ ). The original document length normalization is within each collection (we call each such collection a local collection which corresponds to a text column). If both the length of $D_{\text {Title }}$ and the length of $D_{\text {Lyrics }}$ are equal to the avgdl values (3.21 and 239 as shown in Table 1) in Title and Lyrics respectively, then the $n d l$ values computed by Formula 2.3 are 1 for both documents, while $D_{\text {Lyrics }}$ is more than 70 times longer than that of $D_{\text {Title }}$. However, we desire a smaller weight of a term in the longer document. Therefore, besides the original intra-collection normalization (Formula 2.3), we must consider a new normalization on the average document lengths of local collections (inter-collection document length normalizations). One possible solution might be merging all the collections into a global collection and using a single global avgdl. For the above example, the global avgdl is 116 (see Table 1 for details), and the $n d l$ values for $D_{\text {Title }}$ and $D_{\text {Lyrics }}$ become 0.81 and 1.21 respectively, which are more reasonable values. However, this solution can cause another problem due to the large diversity in avgdl values in different collections. In our example, the global avgdl (116) is dominated by the very long text column
Lyrics (239). Normalizations on short columns are lost as shown in the following new example. Consider the two documents (i.e. two text column values) in tuples $a 1$ and $b 1$ (we call them $D_{a l}$ and $\left.D_{b l}\right)$ as shown in Figure 1. Both $D_{a l}$ and $D_{b l}$ contain the keyword "D12"; the length of $D_{a l}$ is 1 and the length of $D_{b l}$ is 2 . And their $n d l$ values are $0.802^{2}$ and 0.803 respectively. If "D12" occurs in another document whose length is 10 , its $n d l$ is 0.817 . This example shows that this solution fails in the intra document length normalization in the following way: For two different text columns of very different average lengths, which are much shorter than the global avgdl, their $n d l$ values differ very little. However, we desire significant difference between them.

We propose Formula 7 to consider both intra-collection and intercollection document length normalization: (1) we still maintain a local collection for each text column and use Formula 2.3 for intra-collection normalizations, and (2) then we normalize the

$$
n d l=\left((1-s)+s * \frac{d l}{a v g d l}\right) *(1+\ln (a v g d l))
$$

local avgdl for the local collection using $1+\ln (\operatorname{avg} d l)$. This formula can solve the problems shown in the above two examples. The new $n d l$ values computed using Formula 7 for the keyword "how" in a song title document $D_{\text {Title }}$ and a song lyrics document $D_{\text {Lyrics }}$ (suppose their lengths are equal to their own avgdls) become 2.17 and 6.48 respectively $^{3}$. Thus, the term weight in the long document becomes smaller. The new $n d l$ values for the keyword "D12" in $D_{a l}$ and $D_{b l}$ (see Table 1 for their $a v g d l$ values) are $1.48^{4}$ and 1.88 respectively. If "D12" occurs in a document whose length and avgdl are both 10 , the new $n d l$ value is 3.3. They are more reasonable normalizations. Note that this normalization is independent on any keyword in the query.

\subsubsection{Document Frequency Normalization}

Collections have different vocabularies, and term distributions are different. Document frequency normalization also has the problem of local vs. global collections. For example, in the collection of Name of Artist, terms that are people names have high document frequencies. However, document frequencies of these terms are usually low in the collection of Lyrics of Song. In addition, the total numbers of documents in Name of Artist is much smaller than that in Lyrics of Song. Thus, for a term that is a person's name, its idf value in Name of Artist is usually smaller than that in Lyrics of Song. If this term appears in 1/100 of the documents in Name of Artist, and 1/1000 of the documents in Lyrics of Song, then its idf values in the two collections are 4.6 and 6.9 respectively. Furthermore, even in a rather large relational database, some tables may have a small number of tuples, and document frequency statistics in columns of these tables become unreliable. Therefore, we propose to use global document frequency statistics. The $i d f^{g}$ as shown in Formula 8 remains the same as that in Formula 2.2 except we use global statistics, where

$$
i d f^{g}=\ln \frac{N^{g}}{d f^{g}+1}
$$

\footnotetext{
$20.802=(1-s)+s^{*} 1 / 116$, where $s$ is 0.2 , and 116 is the global avgdl. Computations for 0.803 and 0.817 are similar.

$32.17=1 *(1+\ln (3.21))$, and $6.48=1 *(1+\ln (239))$
} 
$d f^{g}$ is the global document frequency of the term (number of documents in the whole database, i.e. all text column values, that the term occurs), and $N^{g}$ is the total number of documents (i.e. the total number of text columns values) in the whole database.

\subsubsection{Inter-Document Weight Normalization}

With the above three normalizations, the term weight in a document $D_{i}$ in $T$ is computed as shown in Formula 5.1, where $n t f$ (normalized term frequency) is computed by Formula 2.1, idf (inverted document frequency) is computed by Formula 8, ndl (normalized document length) is computed by Formula 7, and Nsize(T) (normalized tuple tree size) is computed by Formula 6 . For $\operatorname{Comb}($ ), we can simply sum up the term weights in all of the documents in $T$. However, a term tends to appear more frequently in a $T$ with a larger size. We use Formula 9 to normalize weight $k$,

$$
\operatorname{Comb}()=\max W g t *\left(1+\ln \left(1+\ln \frac{\operatorname{sum} W g t}{\max W g t}\right)\right)
$$

$T$ ), where $\max W g t$ is the maximum weight $\left(k, D_{i}\right)$ and sumWgt is the sum of weight $\left(k, D_{i}\right)$ for all $D_{i}$ in $T$. The idea behind Formula 9 is borrowed from term frequency normalization (Formula 2.1): We consider the maxWgt as one unit of term frequency and sumWgt/max $W g t$ as the total term frequency.

\subsection{Schema Terms in Query}

Another new problem for keyword search in relational databases is that user queries usually contain two types of terms. The first type is terms that are matched with text column values, and we call them value terms. The second type is terms that are matched with the names of text columns, tables and databases, and we call them schema terms. For example, the keyword "lyrics" in Query 2 and the keyword "album" in Query 3 are both schema terms. Schema terms usually do not occur in text values; thus the weight of such a schema term in relevant tuple tress is usually 0 . However, if a text value happens to contain some schema terms, the weights of such schema terms are non-zeroes. One example is a query "lusher the singer's lyrics to burn". There happens to be a song titled "The Singer", and it is ranked top 1 for the query. Obviously, this is not what the user wants. The relevant one should be the song titled "Burn" by the artist "Usher". Although the keyword "singer" matches the name of the attribute Artist, the text column values in this answer do not contain "singer". The weight of "singer" in this answer is 0 , which causes the problem of the relevant answer having a lower similarity value. We use a simple method to solve this problem. For each text column, each table and the database, we identify a set of synonyms that are their names. For example, we generate \{"artist", "band", "singer"\} for the table Artist, \{"album"\} for the table Album, \{"song"\} for the table Song, \{"lyrics"\} for the column Lyrics, etc. For these schema terms, besides their global document frequency values based on their occurrences in text column values, we also assign a schema-based document frequency value for each of them: for a schema term $k$, if it is a synonym of a text column or a synonym of table containing one text column, we assign the largest document frequency value among all terms in the local collection that corresponds to the text column to $k$, and if it is a synonym of a table containing more than one text column, we assign the

\footnotetext{
$41.48=\left((1-s)+s^{*}(1 / 1.89)\right) *(1+\ln (1.89))$, where 1.89 is avgdl of Artist.Name and $\mathrm{s}$ is 0.2 . Computations for 1.88 and 3.3 are similar.
}

largest document frequency value in the collection that corresponds to a union of all the text columns in the table to $k$. Thus, if $k$ is a schema term in a given query, $k$ does not occur in a tuple tree $T$ (i.e., its term frequency is 0 ) but $k$ corresponds to the name of a table or a column involved in $T$, then weight $(k, T)$ computed by Formula 5.2 is 0 . However, we now assume that the term frequency of a schema term $k$ in each of its corresponding text columns involved in $T$ be 1 , and its $d f^{g}$ be its schema-based document frequency. Thus, we can get a weight $(k, T)>0$. If $k$ is a schema term, $k$ occurs in $D_{i}$ of $T$, and $k$ corresponds to the text column of $D_{i}$ or the table containing $D_{i}$, then we compute two values of weight $\left(k, D_{i}\right)$, one is computed by considering $k$ as a value term and the other is computed by considering $k$ as a schema term. Then we choose the larger weight as the final weight $\left(k, D_{i}\right)$ because we assume that each term in the query has only one meaning. For the above example, the final similarity value between the query and the first answer remains unchanged, however the similarity value between the query and the second answer is increased because the term "singer" has a non-zero weight and the second answer becomes top 1 now. The identification of schema terms needs manual work or can be done semi-automatically using WordNet [25]. However, the workload is rather trivial because (1) the number of schema terms is small (only 6 schema terms \{“artist", "band", "singer", "album", "song”, "lyrics"\} are used by users in lyrics search), (2) a well designed database should have information (i.e. schema descriptions) that helps generate these schema terms.

\subsection{Phrase-based Ranking}

Research in IR [14] has shown that phrase-based search can improve effectiveness. Google [15] also utilizes proximity information. In relational databases, many text columns are name entities and favor proximity and phrase search. Consider the subquery "how come" in Query 2. It is a title of a song and should be considered to be a phrase. Another example is "Code Red", a name of an artist (band). Although both keywords are common words, the phrases have specific meanings. To identify phrases from a query, Liu et al. [14] use natural language software and a dictionary to analyze the query. Instead, we only utilize term position information in documents (term position information is stored in the inverted indexes), without using any additional source. Whether a sub-query of a query is a phrase is tuple tree dependent. If a sub-query of $Q, P=\left\{k_{i}, k_{i+1}, . . k_{j}\right\}$, where $i<j$, appears in a document $D$, and $k_{i-1}$ does not appear in an adjacent location to $k_{i}$ in this occurrence of $P$ in $D$, and $k_{j+1}$ does not appear in an adjacent location to $k_{j}$ in this occurrence of $P$ in $D$, then we define it as an occurrence of the phrase $P$ in $D$. We modify Formula 5.1 into Formula 10 to compute weight $(P, D)$, the weight of a phrase $P$ in a document $D$. Since the tuple tree size and document length normalizations are independent to any query keyword, the denominator remains unchanged. The numerator is replaced by $n p f^{*} i d f$. For the $i d f$ component, we assume that $i d f$ of a phrase is the sum of the $i d f s$ of all the individual keywords in the phrase for computational simplicity (Formula 10.2). For $n p f$ (normalized phrase frequency), the same term frequency normalization function (Formula 10.1) is used for a phrase ( $p f$ is phrase frequency in $\left.D_{i}\right)$; in addition, we add a new normalization factor to boost the adjacency of the words in a phrase: $1+\ln (\operatorname{length}(P))$, where length $(P)$ is the number of keywords in $P$. For example, a phrase with two words is 1.7 times more important than the sum of individual term weights when they do not form a phrase. 


$$
\begin{aligned}
& \text { weight }\left(P, D_{i}\right)=\frac{n p f^{*} i d f}{n d l * N \operatorname{size}(T)} \\
& n p f=(1+\ln (1+\ln (p f))) *(1+\ln (\text { length }(P))) \\
& i d f=\sum_{k \in P} \ln \left(\frac{N^{g}}{d f^{g} k+1}\right)
\end{aligned}
$$

The definition of an occurrence of $P$ in $D$ causes a new problem, which is illustrated by the following example. Suppose $Q=\{1,2,3$, $4\}$ and a document $D$ in $T$ is $\{. .1,2,3 \ldots 2,3,4 . .2,3,4 \ldots 1,2 .$. $1 .$.$\} . By the definition, we have phrases \{1,2,3\},\{2,3,4\}$ and $\{1$, $2\}$ in $D$, and their $p f s$ are 1,2 and 1 respectively. However, $\{1,2$, $3\}$ and $\{2,3,4\}$ overlap and neither of them contains the other one. We assume that each occurrence of a phrase or of a keyword in a given query have a unique meaning in a tuple tree. Thus, we only allow one of $\{1,2,3\}$ and $\{2,3,4\}$ to be a phrase for a tuple tree. We propose an algorithm (Figure 7) to re-identify phrases in a document. For example, if the phrase weight of $\{1,2,3\}$ is higher, the output of the algorithm is $\{1,2,3\},\{1,2\}$ and $\{2,3\}$. Their $p f s$ are 1,1 and 2 respectively. If the phrase weight of $\{2,3,4\}$ is higher, the output is $\{2,3,4\}$ and $\{2,3\}$. Their $p f s$ are 2 and 1 respectively. Then we use Formula 10 for phrases and Formula 5.1 for individual keywords to compute the final weight $(Q, T)$.

A phrase may contain shorter phrases or individual keywords, and their weights are computed independently. If we sum them up, we may have a problem that is similar to the inter-document weight normalization problem (Section 4.2.4). For phrases, we slightly modify Formula 9 to borrow the idea of the interdocument weight normalization. When we match a query against documents, we should always do maximal matching, i.e. maximal phrases. For a given query $Q$ and an answer $T$, we first identify the set of phrases and keywords in $T$ that are not contained in any longer phrases. We call this set a concept set $\left(C^{Q}\right)$ in $Q$ against $T$. Then for each concept $c$ (either a phrase or a keyword) in $C^{Q}$, $\max W g t(c)$ is computed as the maximum weight $\left(c, D_{i}\right)$ over all $D_{i}$ in $T$, and $\operatorname{sum} W g t(c)$ is the sum of all weight $\left(p^{\prime}, D_{i}\right)$, where $p^{\prime}$ is $c$, or any shorter phrase or keyword contained in $c$ and $D_{i}$ is a document in $T$. Suppose $c$ has the maximum weight in $D_{i}$, then we (1) use maxWgt(c) as the concept weight of $c$ in $T$, and then (2) bind $c$ to the column of $D_{i}$, (the semantics of the concept $c$ is bound to the text column of $D_{i}$ ). To avoid assigning a column to a single word concept that has a high document frequency and appears in a long document, we set a threshold th. If $\max W g t(c)<t h$, we do not assign the column to $c$. For example, the keyword "by" in Query 2 has a high $d f$ and occurs in a long document (a Lyrics column value in tuple $s 1$ ), and it has a small max Wgt value, thus we do not assign Song.Lyrics to it. However, we assign Artist.Name to the concept "D12" and "Song.Title" to the concept "how come". Thus, a phrase model can return answers with semantics (i.e. the correspondences between concepts in the queries and the columns in the database).

For a query with multiple terms (such as Query 3), a document that contains only some highly weighted terms (due to high idf and/or high term frequencies even we have done $t f$ normalization and/or short document length) may be ranked higher than a document that contains all moderately weighted terms. We want to rank the latter document higher. Our strategy is to use Concept ranking model, which is based on Phrase model. For each concept $c_{i}$ in $C^{Q}$ (described in the above paragraph), we compute a

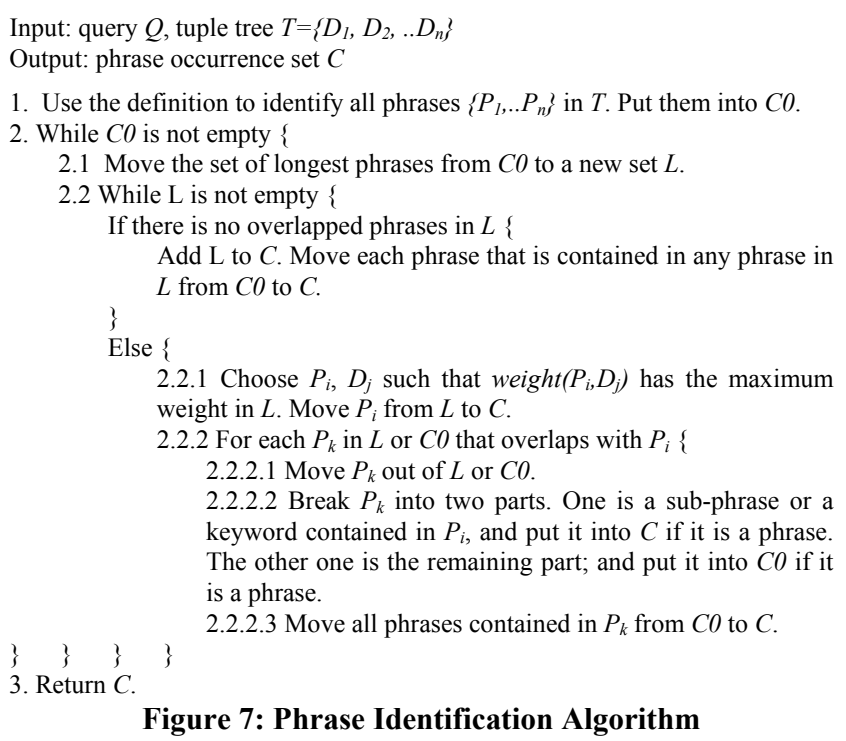

weight $\left(c_{i}, D_{j}\right)$ using Formula 5.1 if $c_{i}$ is a keyword, and using Formula 10 if $c_{i}$ is a phrase. The only exception is that we drop the $1+\ln (1+\ln (t f))$ component from both formulas because we only take into consideration whether a concept appears but not its term frequency according to [14]. Then we choose the maximum weight $\left(c_{i}, D_{j}\right)$ as weight $\left(c_{i}, T\right)$, and sum up all these concept weights to obtain a concept similarity value $\operatorname{Sim}\left(C^{Q}, T\right)$. The similarity between a tuple tree $T$ and a query $Q$ becomes a tuple $\left(\operatorname{Sim}\left(C^{Q}, T\right), \operatorname{Sim}(Q, T)\right)$. The first component is the concept similarity and the second component is the term similarity computed by Formula 5.1 and Formula 9 respectively. For two tuple trees $T_{1}$ and $T_{2}$ and a given query $Q$, if (1) $\operatorname{Sim}\left(C^{Q}, T_{1}\right)>$ $\operatorname{sim}\left(C^{Q}, T_{2}\right)$, or $(2) \operatorname{Sim}\left(C^{Q}, T_{1}\right)=\operatorname{Sim}\left(C^{Q}, T_{2}\right)$ and $\operatorname{Sim}\left(Q, T_{1}\right)>\operatorname{Sim}(Q$, $T_{2}$ ), then $T_{1}$ is ranked higher than $T_{2}$.

\section{EXPERIMENTAL RESULTS}

Section 5.1 describes the data set. Section 5.2 introduces evaluation measures and the setups for comparison. Section 5.3 reports and discusses experimental results on search effectiveness.

\subsection{Data Set}

Database: We use a lyrics database in our experiments. It is reported in [20] that lyrics search is one of the most popular search in various major search engines. We crawled an entire lyrics web site in Aug. 2005, and converted the data into a relational database whose schema is shown in Figure 3. There are more than $177 \mathrm{~K}$ songs in the database. Table 1 gives basic statistics of the database. We do not stem words and do not use stop word except "the".

Query Set: We use a set of 50 queries for evaluation. These queries are obtained from a subset of a one-week period user query $\log$ on a commercial search engine. By using IP addresses and short durations between the times when queries are submitted, we detect that a sequence of queries are submitted by the same users, and each such query is a small variant of the next query in the sequence. By analyzing such queries, the intentions of the users can be found out. This allows us to make relevance assessments of the retrieved results relative to the queries. We randomly choose 50 lyrics queries from the query log (see Appendix for the 50 queries). For each query, we identify a set of 
Table 1: Lyrics Database Statistics

\begin{tabular}{|c|c|c|}
\hline Column & \# of doc & avgdl \\
\hline Artist.Name & 3,691 & 1.89 \\
\hline Album.Title & 15,160 & 2.67 \\
\hline Song.Title & 177,231 & 3.21 \\
\hline Song.Lyrics & 177,231 & 239 \\
\hline
\end{tabular}

relevant answers (tuple trees) in the crawled lyrics database using pooled relevance judgment: we do retrieval using many different methods including Google; for each method, we obtain the top 10 answers and then we get the union of the sets of the top 10 answers; then we judge the relevance for each of the answers in the union. This way of relevant judgment is used in TREC [9]. There are totally 135 relevant answers (2.7 per query on the average). The maximum number of relevant documents for a query is 14 , and the minimum number is 1 . The average length of a query is 6.7 ; the maximum length is 20 and the minimum length is 2 . The average length of such queries is substantially larger than that of typical Internet queries, but in order to specify artists, songs and lyrics, the longer lengths are needed. We further classify them into two sets: simple queries (with only one nonschema concept) and complex queries (with two or more nonschema concepts, and they are underlined in Appendix). Each set contains 25 queries. We expect that our ranking strategy work better on complex queries than on simple queries. We also note that all our queries contain schema terms.

\subsection{Measures and Comparison Setup}

We use 4 measures to evaluate the different aspects of search effectiveness. (1) Number of top-1 answers that are relevant. (2) Reciprocal rank. They measure how good the system is to return one relevant answer. (3) 11-point precision/recall and (4) MAP (see Section 3.1 for Measures 2, 3 and 4). They measure the overall effectiveness for top 10 answers in our experiments (other topk numbers have very similar results). Result comparison serves two purposes: investigating how different factors in our ranking strategy affect search effectiveness, and comparing our ranking strategy with related work to demonstrate its superiority.

The comparison is conducted on three dimensions. (1) Whether the system identifies schema terms (Section 4.3). (2) The four normalization factors described in Section 4.2. (3) The four search models. The first model (All-Word) assumes AND semantics (i.e. an answer must contain all keywords) and ranks answers by number of tuples in the tree in ascending order. The second one (Keyword) assumes OR semantics and uses IR style ranking. But it only considers individual keywords in queries. The third one (Phrase) also considers phrases in queries. The last one (Concept) is a Phrase model that uses concept-based ranking.

\subsection{Results and Discussion on Effectiveness}

\subsubsection{Identification of Schema Terms}

Table 2 gives the reciprocal rank values for all the four models (with all four normalizations) with $(w / s)$ and without $(w / o)$ identification of schema terms. The percentage of improvement (imp) of the run with this factor over the run without this factor is also reported. These values are also reported for complex and simple queries respectively. The results show that identifying schema terms is extremely important for All-Word search model, because it assumes an AND semantics and schema terms are not usually contained in values. This factor seems to have marginal effect on other models (only slight improvement for Concept model on complex queries and Keyword model for all) because in Lyrics database, occurrences of schema terms in values are rare.
Table 2: Identifying Schema Terms

\begin{tabular}{|c|c|c|c|c|c|c|c|c|c|}
\hline \multirow{2}{*}{ Model } & \multicolumn{4}{|c|}{ All } & \multicolumn{3}{c|}{ Complex } & \multicolumn{3}{c|}{ Simple } \\
\cline { 2 - 11 } & w/s & w/o & imp & w/s & w/o & imp & w/s & w/o & imp \\
\hline Concept & 0.871 & 0.850 & $2.5 \%$ & 0.900 & 0.856 & $5.1 \%$ & 0.833 & 0.844 & $-1.3 \%$ \\
\hline Phrase & 0.790 & 0.794 & $-0.5 \%$ & 0.828 & 0.828 & $0.0 \%$ & 0.752 & 0.759 & $-1 \%$ \\
\hline Keyword & 0.696 & 0.662 & $5.1 \%$ & 0.799 & 0.763 & $4.7 \%$ & 0.594 & 0.560 & $6.1 \%$ \\
\hline All-Word & 0.245 & 0.020 & - & 0.316 & 0.000 & - & 0.158 & 0.040 & - \\
\hline
\end{tabular}

Table 3: Tree Size Normalization

\begin{tabular}{|c|c|c|c|c|c|c|c|c|c|}
\hline \multirow{2}{*}{ Model } & \multicolumn{4}{|c|}{ All } & \multicolumn{3}{c|}{ Complex } & \multicolumn{3}{c|}{ Simple } \\
\cline { 2 - 11 } & w/s & w/o & imp & w/s & w/o & imp & w/s & w/o & imp \\
\hline Concept & 0.871 & 0.511 & $70.5 \%$ & 0.900 & 0.178 & $406 \%$ & 0.833 & 0.844 & $-1.3 \%$ \\
\hline Phrase & 0.790 & 0.444 & $77.9 \%$ & 0.828 & 0.129 & $536 \%$ & 0.752 & 0.759 & $-1 \%$ \\
\hline Keyword & 0.696 & 0.357 & $93.3 \%$ & 0.799 & 0.124 & $537 \%$ & 0.594 & 0.589 & $0.8 \%$ \\
\hline
\end{tabular}

Table 4: Document Length Normalizations

\begin{tabular}{|c|c|c|c|c|c|c|c|c|c|}
\hline \multirow{2}{*}{ Model } & \multicolumn{4}{|c|}{ All } & \multicolumn{3}{c|}{ Complex } & \multicolumn{3}{c|}{ Simple } \\
\cline { 2 - 11 } & w/s & w/o & imp & w/s & w/o & imp & w/s & w/o & imp \\
\hline Concept & 0.871 & 0.488 & $78.3 \%$ & 0.900 & 0.395 & $228 \%$ & 0.833 & 0.581 & $42.9 \%$ \\
\hline Phrase & 0.790 & 0.585 & $35.0 \%$ & 0.828 & 0.454 & $82.4 \%$ & 0.752 & 0.715 & $4.9 \%$ \\
\hline Keyword & 0.696 & 0.492 & $40.2 \%$ & 0.799 & 0.477 & $33.0 \%$ & 0.594 & 0.507 & $17.2 \%$ \\
\hline
\end{tabular}

Table 5: Document Frequency Normalization

\begin{tabular}{|c|c|c|c|c|c|c|c|c|c|}
\hline \multirow{2}{*}{ Model } & \multicolumn{4}{|c|}{ All } & \multicolumn{3}{c|}{ Complex } & \multicolumn{3}{c|}{ Simple } \\
\cline { 2 - 11 } & w/s & w/o & imp & w/s & w/o & imp & w/s & w/o & imp \\
\hline Concept & 0.871 & 0.708 & $23.0 \%$ & 0.900 & 0.807 & $11.5 \%$ & 0.833 & 0.600 & $38.8 \%$ \\
\hline Phrase & 0.790 & 0.676 & $16.9 \%$ & 0.828 & 0.779 & $6.3 \%$ & 0.752 & 0.573 & $31.2 \%$ \\
\hline Keyword & 0.696 & 0.685 & $1.6 \%$ & 0.799 & 0.824 & $-3.0 \%$ & 0.594 & 0.548 & $8.4 \%$ \\
\hline
\end{tabular}

Table 6: Inter-Document Weight Normalization

\begin{tabular}{|c|c|c|c|c|c|c|c|c|c|}
\hline \multirow{2}{*}{ Model } & \multicolumn{4}{|c|}{ All } & \multicolumn{3}{c|}{ Complex } & \multicolumn{3}{c|}{ Simple } \\
\cline { 2 - 11 } & w/s & w/o & imp & w/s & w/o & imp & w/s & w/o & imp \\
\hline Concept & 0.871 & 0.871 & $0.0 \%$ & 0.900 & 0.898 & $0.2 \%$ & 0.833 & 0.844 & $-1.3 \%$ \\
\hline Phrase & 0.790 & 0.680 & $16.2 \%$ & 0.828 & 0.756 & $9.5 \%$ & 0.752 & 0.604 & $24.5 \%$ \\
\hline Keyword & 0.696 & 0.591 & $17.8 \%$ & 0.799 & 0.685 & $16.6 \%$ & 0.594 & 0.497 & $19.5 \%$ \\
\hline
\end{tabular}

Table 7: Combining All Normalizations

\begin{tabular}{|c|c|c|c|c|c|c|c|c|c|}
\hline \multirow{2}{*}{ Model } & \multicolumn{3}{|c|}{ All } & \multicolumn{3}{c|}{ Complex } & \multicolumn{3}{c|}{ Simple } \\
\cline { 2 - 11 } & w/s & w/o & imp & w/s & w/o & imp & w/s & w/o & imp \\
\hline Concept & 0.871 & 0.358 & $143.3 \%$ & 0.900 & 0.068 & $1224 \%$ & 0.833 & 0.648 & $28.5 \%$ \\
\hline Phrase & 0.790 & 0.351 & $125.1 \%$ & 0.828 & 0.076 & $989 \%$ & 0.752 & 0.627 & $19.9 \%$ \\
\hline Keyword & 0.696 & 0.248 & $180.6 \%$ & 0.799 & 0.060 & $1232 \%$ & 0.594 & 0.438 & $35.6 \%$ \\
\hline
\end{tabular}

Schema terms rarely form phrases, so it has no effect on Phrase model. In the following sections, all runs use schema terms identification if not otherwise specified.

\subsubsection{Four New Normalizations}

We give four tables (Tables 3-6) to show how each new normalization factor improves keyword search effectiveness using three search models (Keyword, Phrase, and Concept). Table 7 shows how all the new factors improve the overall effectiveness.

Table 3 gives reciprocal rank values for all the three models (with all other three normalizations) with $(w / s)$ tree size normalization and without $(w / o)$ it (but using the simple normalization in Formula 4). The results show that this factor has tremendously significant effect for all three models on complex queries, because the relevant answers $(\operatorname{Size}(T)>1)$ are normalized too much even though we use $(\operatorname{Size}(T)+1) / 2$ instead of $\operatorname{Size}(T)$ [11] in the experiments. It is also not surprising that it has little affect on simple queries, whose answers contain only single tuples and should be ranked higher than all multi-tuple trees.

Table 4 gives reciprocal rank values for all the last three models (with all other three normalizations) with $(w / s)$ our document length normalization and without $(w / o)$ it. The results show that this factor is also very critical for all three models on all queries. 
This factor affects the complex queries more than simple queries because it deals with diverse types (in terms of the document length) of columns. This factor also affects Concept and Phrase models more than Keyword model for complex queries because the first two models boast the weights of long phrases, and longer documents tend to contain more and longer phrases.

Table 5 gives the reciprocal rank values for all the last three models (with all other three normalizations) with $(w / s)$ our document frequency normalization and without $(w / o)$ it. It significantly affects Concept and Phrase models on all queries. It has little effect on Keyword model when all other three normalizations have been applied. The results also show that this factor affects simple queries more than complex queries.

Table 6 gives the reciprocal rank values for all the last three models (with all other three normalizations) with $(w / s)$ our interdocument weight normalization and without $(w / o)$ it. The results show that this factor significantly affects Phrase and Keyword models but less significantly than the tree size and the document length factor. It does not improve effectiveness on Concept model because this model ranks answers with concept similarity in the first order and this factor has no effect on the concept similarity (we always use max $W g t$ ).

Finally, we use Table 7 to demonstrate the overall improvement by comparing models with all the four normalization factors to the same models without any of them. Without any of the four normalizations, all models perform very poorly on complex queries. These normalizations also improve simple queries, but less significantly. We conclude that (1) all these four normalization factors are critical to search effectiveness, (2) the first two factors improve search effectiveness more significantly than the last two, (3) and these normalization affect complex queries more than simple queries.

\subsubsection{Four Models and Related Work}

In Figure 8 and Table 8, Concept, Phrase and Keyword are models that use identification of schema terms with all four normalizations and with OR semantics. Figure 8 shows the 11point precision/recall graph for the four models with all normalizations and with identification of schema terms. MAP values are also given (following the labels). Table 8 also gives results of related work. Concept 2 is the model that does not identify schema terms. Related1 is the All-Word model with identification of schema terms; it is used as an upper bound for the method used in DBXplorer [1] and DISCOVER [10]. Related 2 is the Keyword model with identification of schema terms and without any of the four new normalizations; it is used as an upper bound for the method used in Hristidis et al. [11] with OR semantics. We also report results of Keyworda (i.e. the Keyword model with AND semantics) and Related $2 a$ (i.e. the Related 2 model with AND semantics, which is used as an upper bound for the method in Hristidis et al. [11] with AND semantics). We also compare our results against that obtained by Google. We note again that Google retrieves from text databases instead of relational databases. To evaluate search effectiveness of Google, we submitted each query to Google. Among its top 10 results, we identified the first relevant web page as follows. (1) Based on our relevant assessment, if the relevant answer is a tuple tree with a single tuple, and if a Google result is a web page with the same information as the tuple, then it is relevant. (2) If the relevant

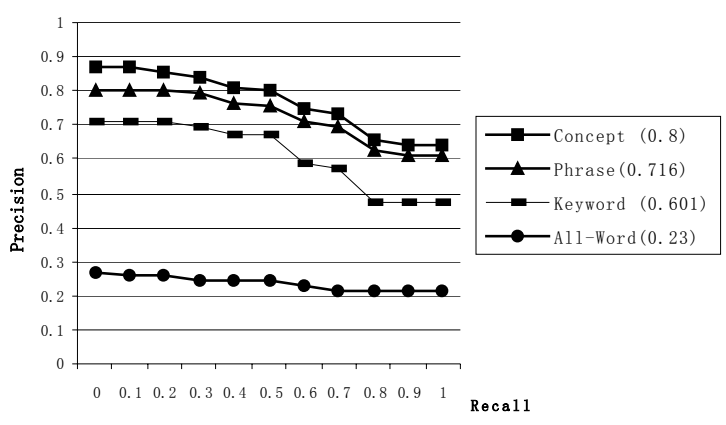

Figure 8: 11-pt Precision/Recall and MAPs for 4 Models

Table 8: Model and Related Work Comparison

\begin{tabular}{|c|c|c|c|c|c|c|}
\hline \multirow{2}{*}{ Model } & \multicolumn{2}{|c|}{ All } & \multicolumn{2}{c|}{ Complex } & \multicolumn{2}{c|}{ Simple } \\
\cline { 2 - 7 } & \# Rel & R-Rank & \# Rel & R-Rank & \# Rel & R-Rank \\
\hline Concept & 39 & 0.871 & 20 & 0.900 & 19 & 0.833 \\
\hline Concept2 & 40 & 0.850 & 20 & 0.856 & 20 & 0.844 \\
\hline Phrase & 35 & 0.790 & 18 & 0.828 & 17 & 0.752 \\
\hline Keyword & 32 & 0.696 & 18 & 0.799 & 14 & 0.594 \\
\hline Keyworda & 29 & 0.615 & 13 & 0.552 & 16 & 0.678 \\
\hline [Related1] & 8 & 0.245 & 6 & 0.316 & 2 & 0.158 \\
\hline [Related2] & 10 & 0.248 & 1 & 0.060 & 9 & 0.438 \\
\hline [Related2a] & 21 & 0.491 & 9 & 0.428 & 12 & 0.554 \\
\hline \hline [Google] & 34 & 0.749 & 15 & 0.709 & 19 & 0.788 \\
\hline
\end{tabular}

answer is a tuple tree with both an Artist tuple and an Album tuple, and if a Google result is a web page with the same information as the Album tuple (this web page usually contains Artist information), then it is relevant. (3) If the relevant answer is a tuple tree with a Song tuple and one or more Artist and/or Album tuples, and if a Google result is a web page with the same information as the Song tuple (this web page usually contains both Album and Artist information), then it is relevant. We do not use the MAP measure in Table 8 due to two reasons: (1) it is highly correlated with the reciprocal rank measure (R-Rank in Table 8) in our experiments, and (2) Google has many copies of lyrics databases from different web sites, and the same answers are often returned multiple times. We also report the number of top-1 answers that are relevant (\#Rel in Table 8).

The results show that Related 11,10$]$ and Related 2 [11] are not as effective as our method. The comparison between Related 2 and Related $2 a^{5}$ shows that, for the ranking method used in Hristidis et al. [11] (Formula 4 in this paper), AND semantics yields significant improvement over OR semantics, especially for complex queries $(0.428$ vs 0.06$)$. The reason is that AND semantics tends to exclude tuple trees containing only one tuple (the ranking scores of these tuple trees using OR semantics are high due to their small size) which contains only a subset of the query keywords (thus they are less relevant). For example, for Related2 (OR semantics) all three relevant answers for the 26th query are ranked below the top 10 results; all the top 10 answers are tuple trees that only contain individual tuples and a subset of the query keywords. For Related2a (AND semantics), most of the top 10 results in Related 2 are excluded because they do not contain all keywords, and among the top 10 results, two are relevant answers that contain multiple tuples. The comparison between Keyword and Keyworda shows that, for our ranking method (Formulas 5.1 and 5.2), OR semantics yields significant

\footnotetext{
${ }^{5}$ For both Related 2 and Related $2 a$, the results of not using schema terms are less effective than those of using schema terms, so they are not reported.
} 
improvement over AND semantics. The comparison between Keyword and Related2, and that between Keyworda and Related $2 a$ show that our ranking method yields significant improvement for both OR semantics (181\%) and AND semantics $(25.3 \%)$ over the ranking method in Hristidis et al. [11]. Concept and Phrase models outperform Google on all queries. Our best result (Concept2) has a 16.3\% improvement over Google and $77.4 \%$ over the best (Related $2 a$ ) of Hristidis et al. [11]. Even when we do not consider schema terms (without any manual work involved), the improvement is $13.5 \%$. From the point view of IR effectiveness, these improvements are significant. For complex queries whose answers are assembled by joining tuples from different tables, all three models outperform Google $(26.9 \%$ for Concept, 20.7\% for Concept 2, 16.8\% for Phrase, and $12.7 \%$ for Keyword) more significantly than for simple queries. And the improvement over Google on simple queries is less significant. We conclude that (1) in terms of search effectiveness, Concept $>$ Phrase $>$ Keyword $>$ All-Word, and (2) our ranking strategy outperforms related work in keyword search in relational databases very significantly and outperforms the state-of-the-art IR method (Google) significantly.

\section{RELATED WORK}

Keyword search in relational databases $[1,2,10,11]$ has recently emerged as a new research topic. DBXplorer [1], DISCOVER [10], Hristidis et al. [11] and BANKS [2] are systems that support free-form keyword search on relational databases. They return tuple trees as answers for a given keyword query. One focus of the above works is to generate tuple trees efficiently. DBXplorer, DISCOVER and Hristidis et al. [1, 10,11] construct a set of join expressions (called answer graph in our paper) for a given query, and then evaluate these join expressions to produce tuple trees. BANKS [2] finds all tuple trees from the data graph directly using a Steiner tree algorithm. In the data graph, they use PageRank style methods to assign weights to tuples and assign weights to edges between tuples. DBXplorer [1], DISCOVER [10] and BANKS [2] assume AND semantics for an answer. To rank answers, they either $[1,10]$ simply use the number of joins in the tuple trees or [2] use a combination of tuple weights and edge weights in a tuple tree without any IR-style ranking method. Hristidis et al. [11] assume OR semantics for answers. To rank answers, they incorporate the IR relevance ranking in a straightforward manner. Many new factors that are critical to search effectiveness are not investigated. Goldman et al. [7] propose a very simple query language with two sets of keywords: Find set and Near set. Two result sets of database objects are obtained for the two sets of keywords. Then the Find result set is re-ranked using distance information between the two sets. Luo et al. [9] combine non-text data values with keyword search on text columns. They concentrate on the efficiency issue of integrating inverted indexes with non-text values. Our work differs from all the above works in three aspects. (1) All of the above works focus on the efficiency issue, not on search effectiveness, which is as important as the efficiency in keyword search, if it is not more important. The only work that mentions effectiveness is BANKS, but they only use 6 queries without any standard evaluation. ObjectRank [3] uses an authority-based ranking strategy for keyword search in relational database. They return individual tuples instead of tuple trees as answers. The ranking score for a tuple is a combination of its ObjectRank value and term frequencies of the query keywords. On effectiveness, they only report non-standard effectiveness measures with two queries. In contrast, we conduct comprehensive experiments using a real database and real user queries with standard evaluation results. (2) Among the above related works, they either do not use IR style ranking at all, or they use it without sufficient consideration of effectiveness (e.g. [11]). In contrast, we propose a novel IR style ranking strategy for the new problem, which is very effective. (3) None returns answers with semantics as we do.

Another different but related research topic is keyword search in XML databases $[6,8,13]$. Florescu et al. [6] extend an XML query language with keyword search. They do not use IR style ranking. XRANK [8] returns XML document fragments as answers. To rank answers, they combine the granularity, hyperlink and keyword proximity information with simple IR style ranking. Both Florescu et al. [6] and XRANK [8] require users to know the XML schema and use a structured query language. $\mathrm{Li}$ et al. [13] propose a schema-free query language for $\mathrm{XML}$, but it is still not structure-free. Kaushik et al. [12] propose an approach to integrating inverted indexes with structured indexes to support more efficient keyword search in XML. Besides the difference between relational and XML databases, none of the above work is for free-form keyword search, and they either incorporate IR-style ranking in a straightforward manner without considering the critical factors in this paper or do not incorporate IR ranking at all. XSEarch [5] proposes a free form keyword query language on XML. To rank answers, which are trees of XML nodes, they combine a simple $t f * i d f$ IR ranking with the size of the tree and the node relationship information. This work only gives examples (but without experimental results) on search effectiveness.

Keyword search has been extensively studied in IR [17, 18, 27] and web search [4, 21]. Sacks-Davis et al. [16] tackle a similar problem with ours from IR's point of view. They investigate indexing methods to support structures in documents. But they do not propose new ranking strategy for the new problem. Recently, link structures [15] have been successfully incorporated with IR ranking in web search. [2, 3, 7, 8] use link structures in the relational or XML databases. The usage of link structures is orthogonal to the usage of IR ranking. We will incorporate link structures in keyword search in our future work.

Major RDBMSs [22, 23, 24] have incorporated IR ranking strategies into their full-text search. However, attribute names must be specified for keywords in SQL queries; free form keyword search is not supported. Our work can be applied into the core of a RDBMS to support free form keyword search (without specifying attribute names). Many web search engines and enterprise search engines are built on structured databases. For example, Google [21] provides product search (froogle), academic paper search (Google scholar), and etc. Amazon.com provides book and product search. Dealtime.com provides product comparison service. IMDB.com has a form-based query interface to search movie information. Although technical details of how they process queries with keywords are absent, experience implies that they do not fully utilize the combination of text data and the database structures. Our work can be applied into these applications to improve search effectiveness.

\section{CONCLUSION AND FUTURE WORK}

Keyword search allows non-expert users to find text information in relational databases with much more flexibilities. In this paper, we proposed a novel ranking strategy for effective keyword 
search in relational databases. A given keyword query is processed in three steps. (1) The system generates all answers (tuple trees) for the query. (2) The system computes a ranking score for each answer and ranks them. (3) Finally, topk answers are returned with semantics.

Our ranking strategy is novel. It identifies and uses four new normalization factors that are critical to search effectiveness: (1) tuple tree size normalization, (2) document length normalization, (3) document frequency normalization and (4) inter-document weight normalization. Schema terms are identified and are processed differently from value terms. Our strategy also uses phrase-based and concept-based models to improve search effectiveness further. And the concept-based model can also return answers with semantics. Comprehensive experiments were conducted using a real world lyrics database and a set of queries collected by a major search engine. Standard evaluation results were reported. The results show that: (1) all the four new normalization factors are critical to search effectiveness (the first two factors improve effectiveness more significantly than the last two, and they improve effectiveness more significantly on complex queries than on simple queries); (2) phrase-based search and concept-based search improve effectiveness significantly; (3) our strategy is significantly better than related works and significantly outperforms Google. Our approach not only can be used at the application level for keyword search in relational databases, but also can be incorporated into the core of a RDBMS.

We plan to utilize link structures (primary key to foreign key relationships as well as some hidden join conditions), and some non-text columns (for example, user review rates on a product, box-office income of a movie, and whether an actor won an Oscar award) in the relational databases along with pure text data. By combining these three pieces of information, we hope to improve search effectiveness further. Furthermore, we plan to investigate the efficiency issue. Finally, we plan to conduct experiments with more real world databases (for example, movie databases, academic paper databases, product databases and job databases) and more user queries.

\section{ACKNOWLEDGEMENTS}

This work is supported in part by NSF grants: IIS-0208434 and IIS-0208574. We thank the anonymous reviewers and Prof. Yannis Papakonstantinou for helpful comments. We thank AOL for providing web search query logs.

\section{REFERENCES}

[1] S Agrawal, S Chaudhuri, G Das: DBXplorer: A system for keywordbased search over relational databases. ICDE 2002

[2] G. Bhalotia, A. Hulgeri, C. Nakhey, S. Chakrabarti, and S. Sudarshan. Keyword searching and browsing in databases using BANKS. ICDE 2002

[3] A. Balmin, V. Hristidis,Y. Papakonstantinou: Authority Based Keyword Queries in Databases using ObjectRank. VLDB 2004

[4] S. Brin and L. Page. The anatomy of a large-scale hypertextual web search engine. WWW 1998

[5] S. Cohen, Jonathan Mamou, Yaron Kanza, Yehoshua Sagiv: XSEarch: A Semantic Search Engine for XML. VLDB 2003

[6] D. Florescu, I. Manolescu, and D. Kossmann. Integrating keyword search into XML query processing. WWW 2000.

[7] R. Goldman, N. Shivakumar, S. Venkatasubramanian, and H. GarciaMolina. Proximity Search in Databases. VLDB 1998.

[8] L. Guo, F. Shao, C. Botev, and J. Shanmugasundaram. XRANK: Ranked keyword search over XML documents. SIGMOD 2003.
[9] L. Guo,J. Shanmugasundaram, K. Beyer, E. Shekita:Efficient Inverted Lists and Query Algorithms for Structured Value Ranking in Update-Intensive Relational Databases. ICDE 2005

[10] V. Hristidis and Y. Papakonstantinou. DISCOVER: Keyword search in relational databases. VLDB 2002.

[11] V. Hristidis,L. Gravano,Y. Papakonstantinou:Efficient IR-Style Keyword Search over Relational Databases.VLDB 2003

[12] R. Kaushik, R. Krishnamurthy, J. Naughton, R. Ramakrishnan. On the integration of structure indexes and inverted lists. SIGMOD 2004

[13] Y. Li, Cong Yu, H. V. Jagadish: Schema-Free XQuery. VLDB 2004

[14] S. Liu, F. Liu, C. Yu, W. Meng: An effective approach to document retrieval via utilizing WordNet and recognizing phrases. SIGIR 2004.

[15] L. Page, S. Brin, R. Motwani, T. Winograd. The PageRank Citation Ranking: Bringing Order to the Web, Technical Report, 1998

[16] R. Sacks-Davis, T. Dao, James A. Thom, Justin Zobel Indexing documents for queries on structure, content and attributes. ISDM97

[17] A. Singhal, Chris Buckley, Mandar Mitra: Pivoted Document Length Normalization. SIGIR 1996

[18] A. Singhal. Modern information retrieval: A brief overview. IEEE Data Eng. Bull. 24(4), 2001

[19] E. M. Voorhees. Overview of the TREC-9 Question Answering Track. TREC 2000

[20] Pew Internet \& American Life Project Report: Search Engine Users, 2005. www.pewinternet.org/pdfs/PIP_Searchengine_users.pd

[21] Google. www.google.com/ 2005

[22] DB2 Text Information Extender. $2005 \mathrm{http} / / / \mathrm{www}-$ 306.ibm.com/software/data/db2/extenders/text/

[23] Micorsoft SQL Server 2000. www.microsoft.com/sq1/ 2005

[24] MySQL. dev.mysql.com/doc/mysq1/en/Fulltext_Search.html.

[25] G. A. Miller. WordNet: A lexical database for English. CACM, 38(11):39--41, 1995.

[26] G. Salton and M. McGill. Introduction to Modern Information Retrieval. McGraw-Hill, 1983

[27] D. Grossman and O. Frieder, Information Retrieval: Algorithms and Heuristics, Springer Publishers, 2nd Edition 2004

\begin{tabular}{|c|c|}
\hline \multicolumn{2}{|c|}{ Appendix: 50 Queries (Complex and Simple) } \\
\hline 1 to me lyrics by lionel richie & 2 inner smile texas lyrics \\
\hline 3 lionel richie lyrics & 4 lionel richie lyrics you mean more to me \\
\hline \multicolumn{2}{|c|}{5 avril lavigne lyrics for the album under this skin } \\
\hline $\begin{array}{l}6 \text { avril lavigne lyrics } \\
8 \text { when i said i do lyrics }\end{array}$ & $\begin{array}{l}7 \text { lyrics to and all } i \text { can taste is this moment } \\
9 \text { when i say i do lyrics }\end{array}$ \\
\hline \multicolumn{2}{|c|}{10 when i say i do lyrics clint black } \\
\hline \multicolumn{2}{|c|}{11 clint black and wife when i say i do lyrics } \\
\hline 12 Hanson i don't know lyrics & 13 lyrics woman's worth \\
\hline 14 lyrics maxwell woman's worth & 15 lyrics maxwell a woman's work \\
\hline 16 lyrics for how come by D12 & 17 usher the singer's lyrics to burn \\
\hline \multirow{2}{*}{\multicolumn{2}{|c|}{$\begin{array}{l}18 \text { lyrics that go "you ran me off the road..you're no longer laughing...i am no } \\
\text { drowning fast enough" } \\
20 \text { lyrics to the songs on simpson's debut album "autobiography" }\end{array}$}} \\
\hline & \\
\hline \multicolumn{2}{|c|}{21 lyrics to rascal flatts moving on 22 lyrics to hanson wheres the love } \\
\hline \multicolumn{2}{|c|}{23 christina millian-dip it low lyrics 24 christina milian-dip it low lyrics } \\
\hline \multicolumn{2}{|c|}{25 Lil Jon "Get Low Remix" lyrics 26 jojo-leave lyrics } \\
\hline \multicolumn{2}{|c|}{27 lyrics to ribbon in the sky 28 slow motion lyrics } \\
\hline 29 i like that lyrics & 30 the way $i$ am lyrics \\
\hline \multicolumn{2}{|c|}{31 i'm just me and thats all i can be lyrics } \\
\hline 32 this yo song ma lyrics & 33 get no better lyrics \\
\hline \multicolumn{2}{|c|}{34 the games have all changed since i been around lyrics } \\
\hline \multicolumn{2}{|c|}{35 cassidy lyrics } \\
\hline \multicolumn{2}{|c|}{36 though i have to find all the answers to my question lyrics } \\
\hline 37 run right through me lyrics & 38 never ever have felt so low lyrics \\
\hline 39 i keep searching lyrics & 40 have you ever felt so low ly \\
\hline \multicolumn{2}{|c|}{41 all the vocabulary runs through my head lyrics } \\
\hline \multirow{2}{*}{\multicolumn{2}{|c|}{$\begin{array}{l}42 \text { lyrics to shania twains song my heart only breaks v } \\
43 \text { lyrics to ashlee simpsons song pieces of me }\end{array}$}} \\
\hline & \\
\hline 44 lyrics to amazed by lonestar & 45 lyrics to I believe by Fantasia \\
\hline \multicolumn{2}{|c|}{46 lyrics to soundtrack to Cradle to the Grave } \\
\hline \multicolumn{2}{|c|}{47 lyrics to focus on the Cradle to the Grave } \\
\hline 48 lyrics to talk about our love by br & \\
\hline 49 edwin mecain lyrics & 0 edwin mccain lyrics better when I'm old \\
\hline
\end{tabular}

